\title{
The VUV/IR/THz Free Electron Laser Program at Jefferson Lab
}

S. V. Benson, J. R. Boyce, D. R. Douglas, P. Evtushenko, F. E. Hannon, C. Hernandez-Garcia, J. M. Klopf, G. R. Neil, M. D. Shinn, C. D. Tennant, S. Zhang and G.P. Williams Jefferson Lab, 12000 Jefferson Avenue, Newport News, VA, 23606, USA

\section{Abstract}

Jefferson Lab operates a pair of oscillator-based continuous-wave Free Electron Lasers (FELs) as a linac-based next generation light source with pulse repetition rates up to $75 \mathrm{MHz}$. The facility uses an energy recovered linac design for efficiency of operation. Recent advances in superconducting technology have been implemented to produce higher acceleration gradients in the linac to produce higher electron beam energies that result in higher photon energies. Thus, while the system originally operated only in the IR, it now covers the photon energy range from the UV to THz, with harmonics upwards of $10 \mathrm{eV}$ with an average spectral flux that is calculated to be $5 \times 10^{17}$ photons/sec/0.1\%BW. Pulse lengths are in the sub-picosecond regime, and the fully coherent nature of the source, both transversely and longitudinally, results in peak and average brightness values that are several orders of magnitude higher than storage rings. The system provides an R\&D test-bed for studies of electron beam dynamics in a regime appropriate for next generation light sources operating at $\mathrm{MHz}$ repetition rates.

\section{Facility Description}

The Jefferson Lab light source facility [1,2] is shown schematically in Fig. 1. It comprises a DC gun-based photoinjector, booster, three linac modules, and two recirculation paths, each containing an oscillator-based FEL comprising an undulator at the center of a 32m optical cavity (round-trip time $4.678 \mathrm{MHz}$ ). The electron and photon beam parameters are given in Table 1, but we note that cryo-cooled mirrors are required to satisfy the higher repetition rates at full power per pulse. We also note that each high power mirror set is designed for high reflectivity and thus has only a few \% tunability. In the present system, except for the cryo-mirrors, we can exchange up to 4 sets of cavity mirrors without breaking vacuum.

Focusing now on the details, in the injector, which is shown at the top left of Fig. 1, electrons are created in bunches by photoemission from a cesiated GaAs wafer, and then accelerated to 10 
$\mathrm{MeV}$ in a booster. They then pass into a linac comprising three JLab cryomodules generating a total of 80-140 MeV of energy gain. The first and third modules incorporate conventional fivecell CEBAF cavities, while the central module is based on new seven-cell JLab cavities [3]. The central module design has demonstrated $82 \mathrm{MV}$ of continuous acceleration, surpassing all previous such systems. In these modules, the electron beam is accelerated (energy recovered) off-crest (off-trough) so as to impose a phase-energy correlation on the longitudinal phase space. This is used in subsequent transport to longitudinally match the beam to the required phase space at the FEL (dump). That is to say, the bunch is kept relatively long during acceleration, compressed to high peak current just before the undulator, then temporally expanded before reinsertion into the energy recovery phase of the linac.

During the electron bunch compression in the in-board magnetic chicane that causes the electron beam to deviate around the first optical cavity mirror, broadband $\mathrm{THz}$ light from collective edge and synchrotron radiation is extracted. From the undulators inside the 32 meter long optical cavities, the electron bunches emit tunable narrow-band light, which is retro-reflected twice so that it can be propagated co-linearly with the electron bunches that follow, to produce gain by longitudinal modulation. At the downstream out-coupling mirror, $10 \%$ of the optical power is extracted with full transverse, as well as longitudinal coherence [4]. This corresponds to a conversion of about $1 \%$ of the electron beam energy. The "spent" electron beam still carrying 99\% of its energy is then re-circulated back into the linac. The energy recovery transport consists of a Bates-style end loop followed by a six-quad telescope [5]. The beam is matched to the arc by the second telescope of the FEL insertion so

51 that the energy recovery telescope matches beam envelopes from the arc to the linac acceptance.

52 Because energy recovery occurs off-trough, the imposed phase-energy correlations are selected

53 to generate energy compression during energy recovery, yielding a temporally long, low

54 momentum spread bunch at the dump. Measurements indicate that the accelerator will tolerate

55 an induced full energy spread from the FEL of as much as 15\% — compressing it to a final spread 56 of order 5\%—despite the large ratio of final to initial energy.

\section{Comparison to other light sources}

58 The light source as described offers photon beams that are many orders of magnitude brighter 59 than storage ring sources. In particular the photon flux is increased by many orders of magnitude 
60 due to phase coherence between wave-fronts emitted by different electrons, i.e. longitudinal, or

61 multiparticle coherent emission [6]. For the 135 pC typical bunch charges used in the Jlab FEL,

62 this enhancement is over 8 orders of magnitude, all of which is realized in the THz beamline [7],

63 and most of which is achieved in the FEL oscillator.

64 In addition to the dramatic increase in photon flux, the brightness of the FEL source is enhanced

65 compared to storage rings by the spatial coherence (small transverse source sizes), which is

66 present in both lateral directions. When electron bunches circulate only once, the electrons can

67 be made to occupy a much tighter 6-dimensional volume of phase-space than in a storage ring,

68 where electron-electron effects and/or quantum excitation spreads them out. In ERL's the

69 horizontal and vertical sizes are essentially the same, rather than the former being 100 times

70 the latter as in storage rings. In our case the rms values of the transverse and longitudinal

71 electron beam sizes are 150 microns. The primary advantage, however, of an ERL driver-

72 based FEL is (in addition to wall-plug efficiency), that of any linac-based source; extremely high

73 longitudinal brightness and the availability of fully coherent light.

74 Major technical advances that enable such a light source to be built and operated are the

75 superconducting linear accelerator structures, which can run cw RF and store energy without

76 significant loss, and photocathode driven electron guns of low emittance.

\section{Scientific Program}

78 With the present FEL, the main interest is in the third harmonic of the UV-FEL which is capable

79 of electronic excitations above the work-function of metals, and above the bandgaps of most

80 insulators. We are in the process of characterizing the third harmonic light, in preparation for its

81 possible utilization for experiments which are summarized in the next 3 paragraphs. Even

82 though the output here is down 2-3 orders of magnitude from the fundamental, light of these

83 photon energies is unobtainable from conventional table-top lasers, and is orders of magnitude

84 brighter than existing storage ring sources, or than High Harmonic Generation (HHG) light [8]

85 see Fig. 2. Additionally the pulses are much shorter than storage ring pulses, and the availability

86 of multiple synchronized photon beams is an added feature that has already been used for novel

87 electro-optic detection [9] leading to measurements of propagating electric fields, with

88 knowledge of phase. Such techniques will bring new dimensions to spectroscopy and imaging,

89 as well as allowing studies of out of equilibrium dynamics. 
90 A unique application of the JLab VUV source is photoemission studies at higher energy and

91 momentum resolution of highly correlated systems such as high $\mathrm{T}_{\mathrm{c}}$ materials [10]. Emitted

92 electron energy and momentum analysis can be done conventionally by dispersion, but can also

93 be done using time of flight techniques, which offer resolution advantages.

94 Another example from Atomic, Molecular and Optical physics, is studies of combustion

95 dynamics [11], as an extension of work already being performed at storage rings. Here the

96 higher brightness and time resolution is used to ionize and study reaction intermediate species of

97 low cross-section in real reaction conditions.

98 A third and final example of a unique scientific application is Atomic Trap Trace Analysis [12],

99 the implementation of the ionization of ${ }^{81} \mathrm{Kr}$ to a metastable state so that it can be used as a tracer

100 isotope for dating water and ice in the range $10^{5}-10^{6}$ years, a range beyond the reach of ${ }^{14} \mathrm{C}$

101 dating and using only liter quantities. This would enable polar ice cores to be dated, for

102 example, using a clock that is significantly longer (229,000 years half-life) than ${ }^{14} \mathrm{C}$ (5730 years).

\section{Potential upgrade path}

104 Increases in electron beam energy from continuing advances in superconducting technology

105

106

107

108

109

110

111

112

113

114

115

116

117

118

119 result in increases in photon energy, which scales as electron beam energy squared. With current designs, cryomodules similar to the ones used in the FEL can achieve $100 \mathrm{MV}$ of acceleration each. Using a recirculating linac scheme, then, with new beam transport around the ends, it is possible to conceive of a $600 \mathrm{MeV}$ electron beam, that with appropriately scaled undulators could make $100 \mathrm{eV}$ light in the fundamental. Such a machine would allow studies of the dynamic structure factor of materials via inelastic scattering. This would open a new door for studying the dynamics of materials in which quasiparticle-quasiparticle interactions dominate physical behavior. Many of these materials, such as the high temperature superconductors, have huge technological potential.

Anticipated performance of such a machine is shown in Fig. 2. An electron beam with a lower normalized emittance of 1 micron, compared with 6 microns at present, would be necessary for this, so we are undertaking studies for such an injector, as well as detailed studies of the beam dynamics of such high brightness electron beams. Issues such as coherent synchrotron radiation (CSR), beam breakup (BBU), and thermal effects in narrow chambers due to high peak currents are examples of issues unique to these sources. The existing machine is a useful test-bed in this 
120 regard. With continuing advances in High Harmonic Generation (HHG) for seed pulses in the 121 range up to $100 \mathrm{eV}$ an amplifier could be designed. However, the high repetition rates possible 122 with superconducting linacs and the use of energy recovery could also enable oscillators. We

123 continue to study these possibilities also, since they could be realized in our existing facility.

\section{Summary}

125 We have described a new generation of light source that started operating part-time as a user 126 facility in 2000, and which, while not funded as a full-time dedicted user facility, has been used 127 continually by users as funds have permitted, with a full proposal and Program Advisory 128 Committee system. The facility was upgraded to the present multi-laser machine configuration 129 in 2010. The machine is based on same-cell energy recovery, a low emittance photo-cathode 130 gun, and a superconducting linac, with each electron making a single trip around. The output of 131 this machine is based almost entirely on multiparticle coherent enhancement which boosts the 132 output photon brightness from the circulating current of $10 \mathrm{~mA}$ up by about 8 orders of 133 magnitude over a storage ring even though the latter may circulate many 100's of mA. Also, it is 134 fair to say that operating experiences with ERL's are different than storage rings. There are 135 different sources of noise, for example, as well as potentially unique opportunities for utilization 136 of multiple photon beams in time-resolved out of equilibrium pump-probe experiments [13]. 137 Even the multiplicity of the user operations is different, but our experiences so far are that our 138 ability to conquer noise, and our cost metrics, are similar to those of storage rings.

\section{Acknowledgements}

140 This work was supported by the Office of Naval Research, the Army Night Vision Laboratory, 141 the Air Force Research Laboratory, the Joint Technology Office, the Commonwealth of Virginia, 142 and by the U.S. DOE Basic Energy Sciences under contract No. DE-AC05-060R23177. 


\begin{tabular}{|c|c|c|}
\hline & IR FEL & UV FEL \\
\hline $\begin{array}{c}\text { Photon energy range of } \\
\text { fundamental }\end{array}$ & $0.1-1.4 \mathrm{eV}$ & $1-3.4 \mathrm{eV}$ \\
\hline Photon energy per pulse & 100 microJoules & 20 microJoules \\
\hline Repetition rate & $4.678-74.85 \mathrm{MHz}$ & $4.678-74.85 \mathrm{MHz}$ \\
\hline Photon Pulse length & $100 \mathrm{fs}-2 \mathrm{ps}$ & $100 \mathrm{fs}-2 \mathrm{ps}$ \\
\hline (FWHM) & & $.2 \%$ \\
\hline Nominal pulse bandwidth & $1 \%$ & $80-140 \mathrm{MeV}$ \\
\hline Electron Beam Energy & $80-140 \mathrm{MeV}$ & 60 picoCoulombs \\
\hline Charge per electron bunch & 135 picoCoulombs & \\
\hline
\end{tabular}

145 


\section{References}

1. G. Neil et al. "Sustained Kilowatt Lasing in a Free-Electron Laser with Same-Cell Energy Recovery”, Phys. Rev. Letts 84, 662 (2000).

2. G. R. Neil et al., “The JLab High Power ERL Light Source” Nucl. Instr. \& Methods A557 9 (2006).

3. I. E. Campisi et al "Superconducting cavity development for the CEBAF upgrade", Proceedings of PAC’99, New York, pp. 937-93929 March-2 April, (1999).

4. S.L. Hulbert and G. P. Williams, "Calculations of Synchrotron Radiation Emission in the Transverse Coherent Limit”, Rev. Sci. Instr. 80, 106103 (2009).

5. J. Flanz and C.P. Sargent, “Operation of an isochronous beam recirculation system”, Nucl. Instr. and Meth. A241 325 (1985).

6. C.J. Hirschmugl, M. Sagurton and G. P. Williams, "Multiparticle Coherence Calculations for Synchrotron Radiation Emission”, Physical Review A44, 1316, (1991).

7. J.M. Klopf et al, "The Jefferson Lab high power THz user facility”, Nucl. Instrum. Methods A582, 114-116 (2007).

8. H.C. Kapteyn, M.M. Murnane, and I.P. Christov, "Extreme Nonlinear Optics: Coherent XRays from Lasers”, Physics Today, 5839 (2005).

9. Y. Cai et al. "Coherent terahertz radiation detection: Direct comparison between free-space electro-optic sampling and antenna detection” Applied Physics Letts. 73, 444 (1998).

10. P. Richard et al., "Nature of oxygen dopant-induced states in high-temperature $\mathrm{Bi}_{2} \mathrm{Sr}_{2} \mathrm{CaCu}_{2} \mathrm{O}_{8+\mathrm{x}}$ superconductors: A photoemission investigation”, Phys Rev B74, 094512 (2006).

11. Craig A. Taatjes, David L. Osborn, Terrill A. Cool, Koichi Nakajima, "Synchrotron Photoionization of Combustion Intermediates: The Photoionization Efficiency of HONO", Chem. Phys. Lett. 394, 19 (2004).

12. X. Du et al., " A new method of measuring ${ }^{81} \mathrm{Kr}$ and ${ }^{85} \mathrm{Kr}$ abundances in environmental samples”, Geophys. Res. Lett. 30, 2068 (2003). 
175 13. G. Lupke, X. Zhang, B. Sun, A. Fraser, N. H. Tolk, and L. C. Feldman, “Structure176 Dependent Vibrational Lifetimes of Hydrogen in Silicon”, Phys. Rev. Lett. 88, 135501, 177 (2002). 
180 Fig. 1. Layout of the JLab FEL facility, showing the injector at the top, the superconducting

181 linac on the upper left, with 2 re-circulation paths each containing an oscillator-based FEL.

182 Fig. 2. Lightsource landscape for average spectral brightness, showing $2^{\text {nd }}, 3^{\text {rd }}$, and $4^{\text {th }}$

183 generation sources, with the JLab FEL operational parameters indicated. These generic plots

184 were made using an electron beam energy of $3 \mathrm{GeV}$, a bending radius of $5 \mathrm{~m}$, and for $1 \mathrm{nC}$

185 electron bunches at $100 \mathrm{MHz}(100 \mathrm{~mA})$. Source sizes used were $500 \times 1000,10 \times 100$, and $5 \times$

1865 microns respectively for the 2,3 and $4^{\text {th }}$ generation sources. $3^{\text {rd }}$ generation sources were

187 elevated by 500 for insertion devices, and $4^{\text {th }}$ generation sources were elevated by a further $10^{10}$

188 to account for longitudinal coherence. 


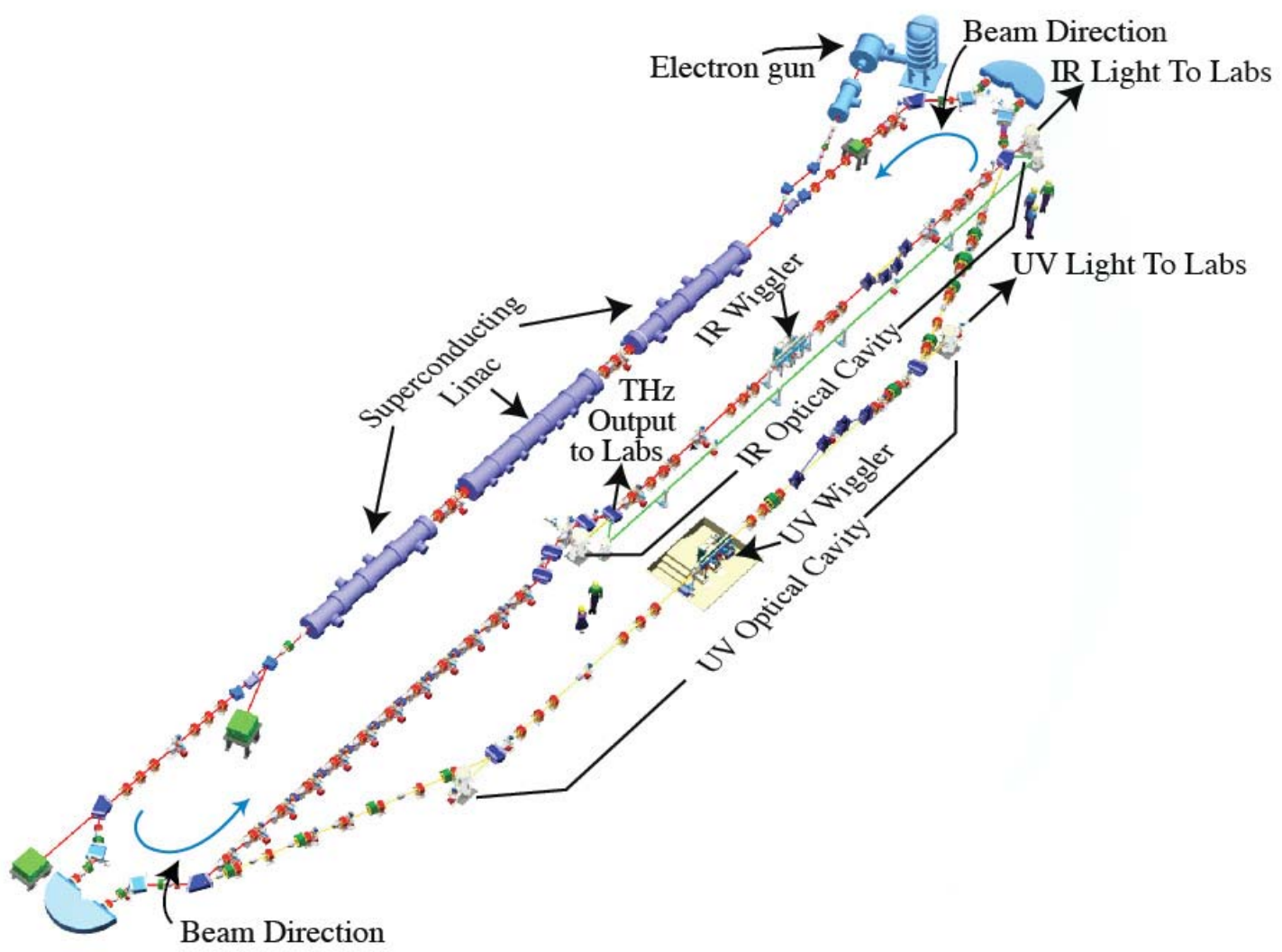

190

191

S. Benson et al. Fig. 1.

192 


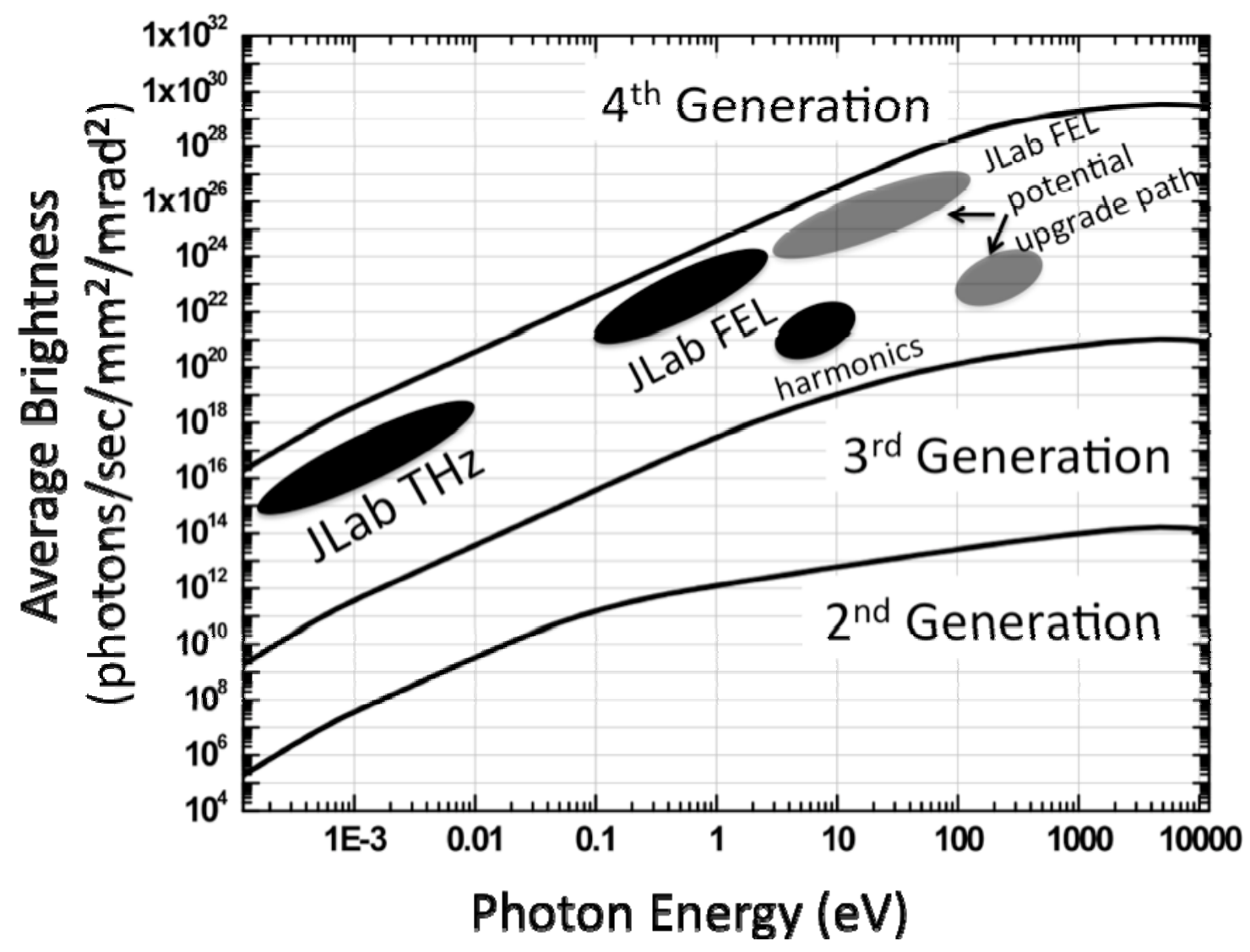

195

S. Benson et al Fig. 2. 